\title{
New Blind Recognition Method of SCLD and OFDM in Alpha-Stable Noise
}

\author{
Junlin Zhang, Bin Wang, Yang Wang \\ National Digital Switching System Engineering and Technological R\&D Centre, Zhengzhou, China \\ Email: commutech@163.com
}

How to cite this paper: Zhang, J.L., Wang, B. and Wang, Y. (2017) New Blind Recognition Method of SCLD and OFDM in Alpha-Stable Noise. Int. J. Communications, Network and System Sciences, 10, 240-251.

https://doi.org/10.4236/ijcns.2017.105B024

Received: April 20, 2017

Accepted: May 23, 2017

Published: May 26, 2017

\begin{abstract}
This paper deals with modulation classification under the alpha-stable noise condition. Our goal is to discriminate orthogonal frequency division multiplexing (OFDM) modulation type from single carrier linear digital (SCLD) modulations in this scenario. Based on the new results concerning the generalized cyclostationarity of these signals in alpha-stable noise which are presented in this paper, we construct new modulation classification features without any priori information of carrier frequency and timing offset of the received signals, and use support vector machine (SVM) as classifier to discriminate OFDM from SCLD. Simulation results show that the recognition accuracy of the proposed algorithm can be up to $95 \%$ when the mix signal to noise ratio (MSNR) is up to $-1 \mathrm{~dB}$.
\end{abstract}

\section{Keywords}

Modulation Recognition, Generalized Second-Order Cyclic Statistics, OFDM, Alpha-Stable Noise

\section{Introduction}

With wide application of OFDM, the recognition of OFDM signal has become a hot issue in various fields of applications among cognitive radio and military applications. Algorithms for the recognition of OFDM versus SCLD signals in AWGN scenario have been reported in [1]-[6]. The algorithm proposed in [1] is based on the signal empirical distribution function to recognize OFDM. Fourthand sixth-order signal moments are employed in [2] to identify OFDM and single-carrier. Wavelet analysis is also employed to distinguish OFDM and SCLD in [3] [4]. The algorithm proposed is based on the second-order cyclic cumulate to recognize OFDM versus SCLD signals under time dispersive channel in [5]. Based on the spectrum analysis, an algorithm was proposed to recognize OFDM in [6]. The above results were developed under the simplified assumption of ad- 
ditive white Gaussian noise channel. But, many noise processes are impulsive in nature. Impulsive channels have been arisen in underwater (ice-cracks), atmospheric (thunderstorms) environments, and other mobile communication problems [7]. Impulsive noise can be modelled as alpha-stable distribution process which is different from Gaussian noise. When the communication channel in nature is impulsive channel modelled as alpha-stable distribution process, the existing recognition algorithms of OFDM versus SCLD would become invalid, because the impulsive noise does not have second-order and higher order statistics. For this reason, we study the recognition of OFDM against SCLD signals in alpha-stable noise here. In this paper, we extract new modulation classification features from the new results concerning the generalized cyclostationarity of OFDM and SCLD signals in alpha-stable noise, and use support vector machine (SVM) as classifier to these signals. The recognition algorithm avoids the need for timing and carrier recovery and the estimation of signal and noise powers. Simulation results show that the proposed method have good performances in alpha-stable noise.

The rest of the paper is organized as follows. The SCLD and OFDM signal models is presented in Sections 2, the proposed recognition algorithm is introduced in Section 3 and simulation results are discussed in Section 4. Finally, our conclusions are presented in Section 5.

\section{Signal Models}

The received signal can be written as,

$$
r(t)=s(t)+w(t)
$$

where $s(t)$ means the transmitted signal, $w(t)$ is alpha-stable noise.

If the modulation type of the transmitted signal is OFDM, $s(t)$ can be represented by $s(t)_{O F D M}$, which is expressed as,

$$
\begin{aligned}
s(t)_{\text {OFDM }} & =A e^{j \theta} e^{j 2 \pi \Delta f_{c} t} \sum_{k=0}^{K-1} \sum_{l-\infty}^{\infty} a_{k, l} e^{j 2 \pi k \Delta f_{k}\left(t-l T_{s}-\mu T_{s}\right)} \\
& \times g\left(t-l T_{s}-\mu T_{s}\right)
\end{aligned}
$$

where $A$ is the amplitude, $\theta$ is the phase, $\Delta f_{c}$ is the carrier frequency offset, $0 \leq \mu \leq 1$ is the timing offset, $g(t)$ is the overall impulse response of the transmit and receive filters, $K$ is the number of subcarriers of the OFDM signal, $a_{k, l}$ is the symbol transmitted on the $k$ th subcarrier over the th symbol period, $\Delta f_{k}$ is the frequency separation between two adjacent subcarriers, $T_{s}$ is the OFDM symbol period, and $T_{s}=T_{u}+T_{c p}$. The data symbols $\left\{a_{k, l}\right\}$ are assumed to be zero-mean independent and identically distributed (i.i.d.) random variables, with values drawn either from a quadrature amplitude modulation (QAM) or phase shift keying (PSK) constellation.

If the modulation type of the transmitted signal is SCLD, $s(t)$ can be represented by $s(t)_{S C L D}$, which is expressed as,

$$
s(t)_{S C L D}=B e^{j \theta} e^{j 2 \pi \Delta f_{c} t} \sum_{l=-\infty}^{\infty} b_{l} g\left(t-l T_{c}-\mu T_{c}\right)
$$


where $B$ is the amplitude, $\theta$ is the carrier phase, $\Delta f_{c}$ is the frequency offset, $T_{c}$ is the symbol period, $0 \leq \mu \leq 1$ is the timing offset, $g(t)$ is the overall impulse response of the transmit and receive filters, $b_{l}$ is the symbol transmitted in the th symbol period. The data symbols $\left\{b_{l}\right\}$ are assumed to be zero-mean independent and i.i.d., with values drawn either from a quadrature amplitude modulation (QAM) or phase shift keying (PSK) constellation.

The alpha-stable noise $w(t)$ is defined by its characteristic function, since a closed form expression for its probability density function (pdf) is not always available. The characteristic function $\phi(u)$ is given by [8],

$$
\phi(u)=\exp \left(j a u-\gamma|u|^{\alpha}[1+j \beta \operatorname{sgn}(u) \omega(u, \alpha)]\right)
$$

In (4), $\omega(u, \alpha)$ and $\operatorname{sgn}(u)$ is given as,

$$
\omega(u, \alpha)=\left\{\begin{array}{rr}
\tan (\pi \alpha / 2) & \alpha \neq 1 \\
(2 / \pi) \log |u| & \alpha=1
\end{array}\right.
$$

and

$$
\operatorname{sgn}(u)=\left\{\begin{array}{rr}
1 & u>0 \\
0 & u=0 \\
-1 & u<0
\end{array}\right.
$$

where $\alpha, \gamma$, and $a$ are the characteristic exponent, dispersion, and location, respectively. Without loss of generality, noise employs standard symmetric alpha-stable distribution-SaS as model. Because the SaS noise do not have finite second moment, its noise variance loses meaning. So a mixed signal to noise ratio-MSNR is employed, MSNR is given by [8],

$$
M S N R=10 \lg \left(\sigma_{s}^{2} / \gamma\right)
$$

where $\sigma_{s}^{2}$ represents the signal variance and $\gamma$ is dispersion of alpha-stable noise.

\section{The Recognition Algorithm of OFDM and SCLD in Alpha-Stable Distributed Noise}

\subsection{The Generalized Second-Order Cyclic Statistics of OFDM and SCLD}

Alpha-stable noise does not have second-order and higher order statistics so that many traditional OFDM identification algorithms will be invalid. To solve the problem that traditional second-order cyclic statistics significantly degenerate in the alpha-stable noise, the concept of generalized second-order cyclic statistics was proposed in [9]. The core of the concept is by use of some kind of nonlinear transformation to suppress the pulse noise, and then process the transformed signal by traditional second-order cyclic statistical analysis. Taking advantages of the concept, a new method to recognize modulation type between OFDM and SCLD in this paper. Firstly, a nonlinear transformation function is defined as following to transform the received signal 


$$
f[r(t)]=\frac{1}{|r(t)|} r(t)
$$

where $r(t)$ represents the received signal.

The autocorrelation function of signal $f[r(t)]$ can be expressed as,

$$
\begin{aligned}
G(t, \tau) & =E\left[f[r(t)] f^{*}[r(t+\tau)]\right] \\
& =E\left[\frac{s(t) s^{*}(t+\tau)}{|s(t)+w(t)||s(t+\tau)+w(t+\tau)|}\right. \\
& \left.+\frac{w(t) w^{*}(t+\tau)}{|s(t)+w(t)||s(t+\tau)+w(t+\tau)|}\right]
\end{aligned}
$$

When MSNR is higher enough, $|s(t)| \gg|w(t)|$, (9) can be approximated as,

$$
\begin{aligned}
G(t, \tau) & =E\left[f[r(t)] f^{*}[r(t+\tau)]\right] \\
& \approx E\left[\frac{s(t) s^{*}(t+\tau)}{|s(t)||s(t+\tau)|}\right]+E\left[\frac{w(t) w^{*}(t+\tau)}{|s(t)||s(t+\tau)|}\right] \\
& =G_{s}(t, \tau)+G_{w}(t, \tau)
\end{aligned}
$$

where $G_{s}(t, \tau)=E\left[\frac{s(t) s^{*}(t+\tau)}{|s(t)||s(t+\tau)|}\right]$,

$$
G_{w}(t, \tau)=E\left[\frac{w(t) w^{*}(t+\tau)}{|s(t)| s(t+\tau) \mid}\right], G_{s}(t, \tau) \text { represents autocorrelation information }
$$

of $s(t)$. When $|s(t)| \gg|w(t)|$, the amplitude of noise is suppressed. Obviously, when processing the received signal $r(t)$ with nonlinear transformation, the unique features of signal $s(t)$ are maintained in $G_{s}(t, \tau)$, and the amplitude of noise is effectively limited. For the OFDM signal, (2) can be put into (10) and (10) becomes

$$
\begin{aligned}
& G_{\text {OFDM }}(t, \tau)=E\left[\frac{s(t) s^{*}(t+\tau)}{|s(t)| \cdot|s(t+\tau)|}\right] \\
& =E\left[\frac{e^{-j 2 \pi \Delta f_{c} t} \sum_{k=0}^{K-1} a_{k}(t) e^{j 2 \pi k \Delta f_{k} t} g(t)}{\left|\sum_{k=0}^{K-1} a_{k}(t) e^{j 2 \pi k \Delta f_{k} t} g(t)\right|}\right. \\
& \left.\times \frac{\sum_{k=0}^{K-1} a_{k}^{*}(t+\tau) e^{-j 2 \pi k \Delta f_{k}(t+\tau)} g^{*}(t+\tau)}{\left|\sum_{k=0}^{K-1} a_{k}(t+\tau) e^{j 2 \pi k \Delta f_{k}(t+\tau)} g(t+\tau)\right|}\right] \otimes \sum_{l=-\infty}^{\infty} \delta\left(t-l T_{s}-\mu T_{s}\right) \\
& =E\left[\frac{e^{-j 2 \pi \Delta f_{c} \tau} \sum_{k=0}^{K-1} a_{k}(t) e^{j 2 \pi k \Delta \Delta_{k} t} \sum_{k=0}^{K-1} a_{k}^{*}(t+\tau) e^{-j 2 \pi k \Delta f_{k}(t+\tau)}}{\left|\sum_{k=0}^{K-1} a_{k}(t) e^{j 2 \pi k \Delta f_{k} t}\right|\left|\sum_{k=0}^{K-1} a_{k}(t+\tau) e^{j 2 \pi k \Delta f_{k}(t+\tau)}\right|}\right] \\
& \times P[g(t)] P\left[g^{*}(t+\tau)\right] \otimes \sum_{l=-\infty}^{\infty} \delta\left(t-l T_{s}-\mu T_{s}\right)
\end{aligned}
$$

where $a_{k}(t)$ is the information data transmitted within the $k$ th subcarrier, and 
the data is assumed to be i.i.d.. Hence, (11) can be further re-written as,

$$
\begin{aligned}
G_{\text {OFDM }}(t, \tau) & =E\left[\frac{s(t) s^{*}(t+\tau)}{|s(t)||s(t+\tau)|}\right] \\
& =\left(e^{-j 2 \pi \Delta f_{c} \tau} \sum_{k=0}^{K-1} C_{k, s}(t) e^{-j 2 \pi k \Delta \Delta_{k} \tau} P[g(t)]\right. \\
& \left.\times P\left[g^{*}(t+\tau)\right]\right) \otimes \sum_{l=-\infty}^{\infty} \delta\left(t-l T_{s}-\mu T_{s}\right)
\end{aligned}
$$

where $C_{s}(t)=E\left[\frac{a_{k}(t) a_{k}(t+\tau)}{\bar{s}(t) \bar{s}(t+\tau)}\right] \geq 0$,

$$
\bar{s}(t)=\left|\sum_{k=0}^{K-1} a_{k}(t) e^{j 2 \pi k \Delta f_{k} t}\right|,(\cdot)^{*} \text { represents the complex conjugate, } P[\cdot] \text { stand }
$$

for operator, which maintain the phase of signal, $\delta(\cdot)$ is the Dirac delta function and $\otimes$ is convolution. From (12) one can see that $G_{\text {OFDM }}(t, \tau)$ is a periodic function with fundamental periodic $T_{s}$. The autocorrelation function can be easily expressed as a Fourier series, and the Fourier transform of (12) yields

$$
\begin{aligned}
& G_{\text {OFDM }}(\varepsilon, \tau)=\mathfrak{J}\left\{G_{\text {OFDM }}(t, \tau)\right\} \\
& =\int_{-\infty}^{\infty}\left(e^{-j 2 \pi \Delta f_{c} \tau} \sum_{k=0}^{K-1} C_{s}(t) e^{-j 2 \pi k \Delta f_{k} \tau} P[g(t)]\right. \\
& \left.\times P\left[g^{*}(t+\tau)\right]\right) \otimes \sum_{l=-\infty}^{\infty} \delta\left(t-l T_{s}-\mu T_{s}\right) e^{-j 2 \pi t \varepsilon} d t
\end{aligned}
$$

where $\mathfrak{I}\{\cdot\}$ denotes the Fourier transform. Furthermore, by expressing the convolution, using a change of variables and the identity

$$
\begin{aligned}
\mathfrak{J}\left[\sum_{l-\infty}^{\infty} \delta\left(t-l T_{s}\right)\right]= & \frac{1}{T} \sum_{l=-\infty}^{\infty} \delta\left(\varepsilon-l T_{s}^{-1}\right), \text { one can show that (13) becomes } \\
& G_{\text {OFDM }}(\varepsilon, \tau) \\
& =\int_{-\infty}^{\infty}\left(e^{-j 2 \pi \Delta f_{c} \tau} \sum_{k=0}^{K-1} C_{s}(t) e^{-j 2 \pi k \Delta f_{k} \tau} P[g(t)] P\left[g^{*}(t+\tau)\right]\right) \\
& \otimes \sum_{l-\infty}^{\infty} \delta\left(t-l T_{s}-\mu T\right) e^{-j 2 \pi t \varepsilon} d t \\
& =\frac{1}{T_{s}} e^{-j 2 \pi \Delta f_{c} \tau} e^{-j 2 \pi \varepsilon \mu T_{s}} \sum_{k=0}^{K-1} e^{-j 2 \pi k \Delta f_{k} \tau} \int_{-\infty}^{+\infty} C_{s}(t) P[g(t)] \\
& \times P\left[g^{*}(t+\tau)\right] e^{-j 2 \pi t \varepsilon} d t \sum_{l=-\infty}^{+\infty} \delta\left(\varepsilon-l T_{s}^{-1}\right) \\
& =\left\{\begin{array}{l}
\frac{1}{T_{s}} e^{-j 2 \pi \Delta f_{c} \tau} e^{-j 2 \pi \varepsilon \mu T_{s}} \sum_{k=0}^{K-1} e^{-j 2 \pi k \Delta f_{k} \tau} \int_{-\infty}^{+\infty} C_{s}(t) \\
\times P[g(t)] P\left[g^{*}(t+\tau)\right] e^{-j 2 \pi t \varepsilon} d t \quad \varepsilon=l T_{s}^{-1} \\
0
\end{array}\right.
\end{aligned}
$$

where $\Xi_{k}(\tau)=\sum_{k=0}^{K-1} e^{-j 2 \pi k \Delta f_{k} \tau}=e^{j \pi(1-K) \Delta f_{k} \tau} \frac{\sin \left(\pi K \Delta f_{k} \tau\right)}{\sin \left(\pi \Delta f_{k} \tau\right)}$. Note that (14) give the analytical expressions for the generalized second-order cyclic statistics at $\varepsilon=l T_{s}^{-1}$ and certain delay. According to (14), when $\tau=1 / \Delta f_{K}=T_{u}, \Xi_{k}(\tau)$ 
yields significant peaks. Meanwhile, the magnitude peaks of $G_{\text {OFDM }}(\varepsilon, \tau)$ are visible at $\tau=T_{u}$ and $\varepsilon=l T_{s}^{-1}$, with $l$ as an integer.

As one can notice, when processing the received signal $r(t)$ with nonlinear transformation, the modulated information of received signal $r(t)$ is maintained in the $G_{\text {OFDM }}(t, \tau)$. For the OFDM signal, significant peaks occur in the vicinity of $\tau=T_{u}$ and $\varepsilon=l T_{s}^{-1} . \mathfrak{J}\left\{G_{w}(t, \tau)\right\}$ stands for noise interference, and its impact has been suppressed due to the nonlinear transformation.

Similarly, for the SCLD signal, we obtain

$$
\begin{aligned}
G_{S C L D}(\varepsilon, \tau) & =T_{c}^{-1} e^{-j 2 \pi \Delta f_{c} \tau} e^{-j 2 \pi \varepsilon \mu T_{c}} \int_{-\infty}^{\infty} b(t) P[g(t)] \\
& \times P\left[g^{*}(t+\tau)\right] e^{-j 2 \pi t \varepsilon} d t \sum_{l=-\infty}^{+\infty} \delta\left(\varepsilon-l T_{c}^{-1}\right)
\end{aligned}
$$

where $b(t)$ is information data transmitted and the data is assumed to be i.i.d.. According to (15), the magnitude peaks of $G_{S C L D}(\varepsilon, \tau)$ are visible at $\tau=0$ and $\varepsilon=l T_{c}^{-1}$.

\subsection{The Generalized Cyclostationarity-Based Recognition Method of OFDM and SCLD Signals}

Based on the above results on signal the generalized cyclic statistics, here we develop a novel algorithm for the recognition of OFDM and SCLD signals in alpha-stable noise.

Under the assumption of no aliasing, for the discrete-time signal $r(n)=\left.r(t)\right|_{n=t t_{s}^{-1}}$, obtained by sampling the continuous-time signals $r(t)$ at the sampling rate $f_{s}$. According to the discussed in Section 3.1, we obtain

$$
\begin{aligned}
G(n, \tilde{\tau}) & =E\left[f[r(n)] \cdot f^{*}[r(n+\tilde{\tau})]\right] \\
& \approx E\left[\frac{s(n) s^{*}(n+\tilde{\tau})}{|s(n)||s(n+\tilde{\tau})|}\right]+E\left[\frac{w(n) w^{*}(n+\tilde{\tau})}{|s(n)||s(n+\tilde{\tau})|}\right] \\
& =G_{s}(n, \tilde{\tau})+G_{w}(n, \tilde{\tau})
\end{aligned}
$$

Then, for the OFDM signal, $G_{s}(n, \tilde{\tau})$ can be written as

$$
\begin{aligned}
& G_{\text {OFDM }}(n, \tilde{\tau})=\left(e^{-j 2 \pi \Delta f_{c} \tilde{\tau} f_{s}^{-1}} \sum_{k=0}^{K-1} C_{s}(n) e^{-j 2 \pi k \Delta f_{k} \tilde{\tau} f_{s}^{-1}}\right. \\
& \left.\times P[g(n)] \times P\left[g^{*}(n+\tilde{\tau})\right]\right) \otimes \sum_{l=-\infty}^{\infty} \delta\left(n-l N_{s}-\mu N_{s}\right)
\end{aligned}
$$

where $C_{s}(n)=E\left[\frac{a_{k}(n) a_{k}(n+\tilde{\tau})}{\bar{s}(n) \bar{s}(n+\tilde{\tau})}\right] \geq 0$,

$$
\bar{s}(n)=\left|\sum_{k=0}^{K-1} a_{k}(n) e^{j 2 \pi k \Delta f_{k} n}\right|, \quad N_{s} \text { denotes OFDM symbol length, and }
$$
$N_{s}=N_{u}+N_{c p}, \quad N_{u}=f_{s} / \Delta f_{K}$ is the useful symbol length and $N_{c p}$ is the cyclic prefix length. From (17) one can see that $G_{\text {OFDM }}(n, \tilde{\tau})$ is a periodic function, with fundamental periodic $N_{s}$. Thus $G_{\text {OFDM }}(n, \tilde{\tau})$ can be expressed as a Fourier series, and the Fourier transform of (17) yields 


$$
\begin{aligned}
& G_{O F D M}(v, \tilde{\tau})=\mathfrak{I}\left\{G_{O F D M}(n, \tilde{\tau})\right\} \\
& =\sum_{n}\left(e^{-j 2 \pi \Delta f_{c} \tilde{\tau} f_{s}^{-1}} \sum_{k=0}^{K-1} C_{s}(n) e^{-j 2 \pi k \Delta f_{k} \tilde{\tau} f_{s}^{-1}} P[g(n)]\right. \\
& \left.\times P\left[g^{*}(n+\tilde{\tau})\right]\right) \otimes \sum_{l=-\infty}^{\infty} \delta\left(n-l N_{s}-n_{0}\right) e^{-j 2 \pi v n} \\
& =\frac{1}{N_{s}} e^{-j 2 \pi v \mu N_{s}} e^{-j 2 \pi \Delta f_{c} \tilde{\tau} f_{s}^{-1}} \sum_{n} \sum_{k=0}^{K-1} e^{-j 2 \pi k \Delta f_{k} \tilde{\tau} f_{s}^{-1}} C_{s}(n) \\
& \times P[g(n)] P\left[g^{*}(n+\tilde{\tau})\right] e^{-j 2 \pi v n} \sum_{l=-\infty}^{+\infty} \delta\left(v-l N_{s}^{-1}\right)
\end{aligned}
$$

where $\Xi_{k}(\tilde{\tau})=\sum_{k=0}^{K-1} e^{-j 2 \pi k \Delta f_{k} \tilde{\tau} f_{s}^{-1}}=e^{j \pi(1-K) \Delta f_{k} \tilde{\tau} f_{s}^{-1}} \frac{\sin \left(\pi K \Delta f_{k} \tilde{\tau} f_{s}^{-1}\right)}{\sin \left(\pi \Delta f_{k} \tilde{\tau} f_{s}^{-1}\right)}$, the significant peaks of $\Xi_{k}(\tilde{\tau})$ can be obtained at $\tilde{\tau}=f_{s} / \Delta f_{K}=N_{u}$. Furthermore, from (18) it can be noticed that magnitude peaks are visible at $\tilde{\tau}=N_{u}$ and $v=l / N_{s}^{-1}$, with $l$ as an integer.

Similarly, for the SCLD signal, we obtain

$$
\begin{aligned}
G_{S C L D}(v, \tilde{\tau}) & =\mathfrak{J}\left\{G_{S C L D}(n, \tilde{\tau})\right\} \\
& =N_{c}^{-1} e^{-j 2 \pi \Delta f_{c} \tilde{\tau} f_{s}^{-1}} e^{-j 2 \pi v \mu N_{c}} \sum_{n}(b(n) P[g(n)] \\
& \left.\times P\left[g^{*}(n+\tilde{\tau})\right]\right) e^{-j 2 \pi v n} \sum_{l=-\infty}^{+\infty} \delta\left(v-l N_{c}^{-1}\right)
\end{aligned}
$$

where $C(n)=E\left[\frac{b(n) b(n+\tilde{\tau})}{|b(n)||b(n+\tilde{\tau})|}\right] \geq 0, \quad b(n)$ is information of data transmitted and the data is assumed to be independent and identically distributed. $N_{c}$ denotes symbol length of SCLD. According to (19), magnitude peaks of are visible at $\tilde{\tau}=0$ and $v=l N_{c}^{-1}$, with $l$ as an integer.

When comparing (18) and (19), one can easily notice the additional factor $\Xi_{k}(\tilde{\tau})$ which appears in the expression of the generalized cyclic statistics of the OFDM signal. One can easily show that significant peaks of the generalized cyclic statistics of OFDM signal yields at $\tilde{\tau}=0$ and $v=l N_{c}^{-1}$. The existence of such a peak in the magnitude of the generalized cyclic statistics of the OFDM signal (at $\tilde{\tau}=N_{u}$ and $v=0$ ) is employed as discriminating feature to identify OFDM against SCLD signals.

The estimate of the generalized cyclic statistics $\hat{G}(v, \tilde{\tau})$ exist the estimation error factor which vanishes asymptotically is $N \rightarrow \infty$. Due to the existence of estimation error factor, the estimator seldom exactly zero in practice, even if $v$ is not at generalized cycle frequencies. In order to decrease the impact of estimation error factor, referencing [10], the modulation classification features are given as,

$$
\Psi=W \hat{C}_{r}^{v}\left(\hat{\Sigma}_{r}\right)^{-1}\left(\hat{C}_{r}^{v}\right)^{\dagger}
$$

where $\hat{C}_{r}^{v}=[\operatorname{Re}\{\hat{G}(v, \tilde{\tau})\} \quad \operatorname{Im}\{\hat{G}(v, \tilde{\tau})\}], \quad v \quad$ is tested candidate frequency and $\tilde{\tau}$ represents tested candidate delay, $W$ is the received signal length. Here $\dagger$ and $(\cdot)^{-1}$ denote transpose and matrix inverse, $\hat{\Sigma}_{r}$ is an estimate of 
the covariance matrix

$$
\hat{\Sigma}_{r}=\left[\begin{array}{ll}
\operatorname{Re}\left\{\left(Q_{20}+Q_{21}\right) / 2\right\} & \operatorname{Im}\left\{\left(Q_{20}-Q_{21}\right) / 2\right\} \\
\operatorname{Im}\left\{\left(Q_{20}+Q_{21}\right) / 2\right\} & \operatorname{Re}\left\{\left(Q_{21}-Q_{20}\right) / 2\right\}
\end{array}\right]
$$

with covariance estimators are respectively given by,

$$
\begin{aligned}
Q_{2,1} & =\left(W \ell_{w}\right)^{-1} \sum_{v=-\left(\ell_{w}-1\right) / 2}^{\left(\ell_{w}-1\right) / 2} \omega(v) F^{*}\left(2 \pi v+2 \pi v W^{-1}\right) \\
& \times F\left(2 \pi v+2 \pi v W^{-1}\right)
\end{aligned}
$$

and

$$
\begin{aligned}
Q_{2,0}= & \left(W \ell_{w}\right)^{-1} \sum_{v=-\left(\ell_{w}-1\right) / 2}^{\left(\ell_{w}-1\right) / 2} \omega(v) F\left(2 \pi v-2 \pi v W^{-1}\right) \\
& \times F\left(2 \pi v+2 \pi v W^{-1}\right)
\end{aligned}
$$

where $F(v)=\sum_{w=0}^{W-1} r(w+\tilde{\tau}) r^{*}(I) e^{-j 2 \pi v w}, \omega(v)$ is a spectral window of support $\ell_{w}$ (odd).

According to the above analysis, the steps of recognizing OFDM against SCLD modulations may be obtained as follows:

Step 1: The magnitude of the generalized second-order cyclic statistics $\hat{G}(v, \tilde{\tau})$ of the received signal is estimated. Firstly, the discrete-time signal $r(n)$ obtained by sampling the continuous-time signal $r(t)$ at the sampling rate $f_{s}$. Then, the nonlinear transformation function in (8) is used to map received signal $r(n)$. Finally, the normalization magnitude of the second-order cyclic statistics $\hat{G}(v, \tilde{\tau})$ of the transformed signal $f[r(t)]$ is estimated.

Step 2: The modulation classification features $\Psi$ is extracted from $\hat{G}(v, \tilde{\tau})$. Search the maximum $\hat{G}(0, \tilde{\tau})$ value at $v=0$ section, and the delays $\tilde{\tau}$ in the interval $\left[\rho K_{\min }, \rho K_{\max }\right]$ (with and as the minimum and maximum number of subcarriers that we consider). Then, the modulation classification features $\Psi$ is extracted with (20).

Step 3: The modulation type is decided. Classify the test signal by employing the SVM classifier defined in the training procedure.

\section{Simulation Results and Analysis}

\section{A. Simulation setup}

For the SCLD signals, we consider a pool of QPSK and 16-QAM. With the OFDM signals, the subcarriers are modulated using QPSK or 16-QAM. Unless otherwise mentioned, for the OFDM signal, the number of subcarriers is set to 64 , the useful time period of the OFDM symbol is set to $0.0128 \mathrm{~s}$ and the cyclic prefix period is set to $0.0032 \mathrm{~s}$. In addition, frequency offset is set to $1 \mathrm{kHz}$ and 2 $\mathrm{kHz}$ for SCLD and OFDM signals, and $\mu$ to 0.75 , as $\theta$ a random variable uniformly distributed over $[-\pi, \pi)$. The SVM classifier is implemented by utilizing the LIBSVM toolbox, where the Gaussian RBF is selected as the kernel function. Let $H_{i}$ be the training sequence composed of $\Psi$ at the different MSNR, where $i \in\{1, \cdots, V\}$, with $V$ as the number of the training sequences. 
The number of training and test sequences is respectively set to 100 for each class at each MSNR.

B. Generalized second-order cyclic statistics magnitude estimates for the

\section{OFDM and SCLD signals}

The estimated magnitude of the generalized cyclic statistics of OFDM and SCLD is plotted. For SaS noise, MSNR $=10 \mathrm{~dB}$ and $\alpha=1.5$.

When comparing results presented in Figure 1 and Figure 2, one can notice that the second-order cyclic spectrum of OFDM signal is destroyed by alpha-stable noise, but the generalized second-order cyclic statistics of OFDM signal yields significant peaks at $\tilde{\tau}=N_{u}$ and $v=l / N_{s}^{-1}$. Figure 3 shows that the generalized second-order cyclic statistics of SCLD signal can keep the characteristics of second-order cyclic statistics. However, Figure 4 shows that the second-order cyclic statistics of SCLD signal is destroyed by alpha-stable noise. From Figure 1 and Figure 3 one can notice that the existence of such a peak in

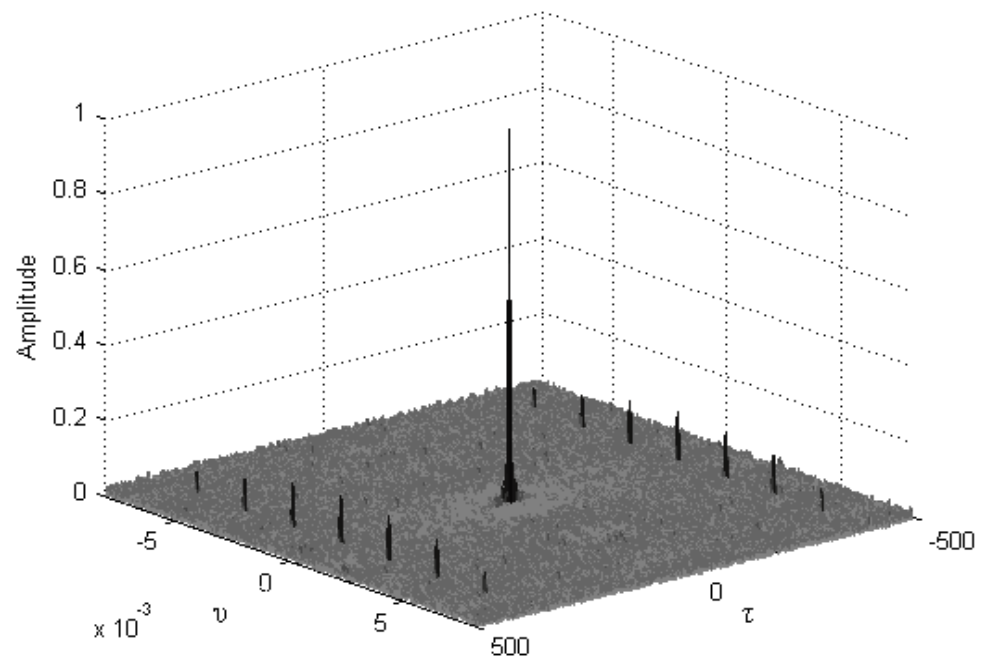

Figure 1. Generalized second-order cyclic statistics of OFDM signal.

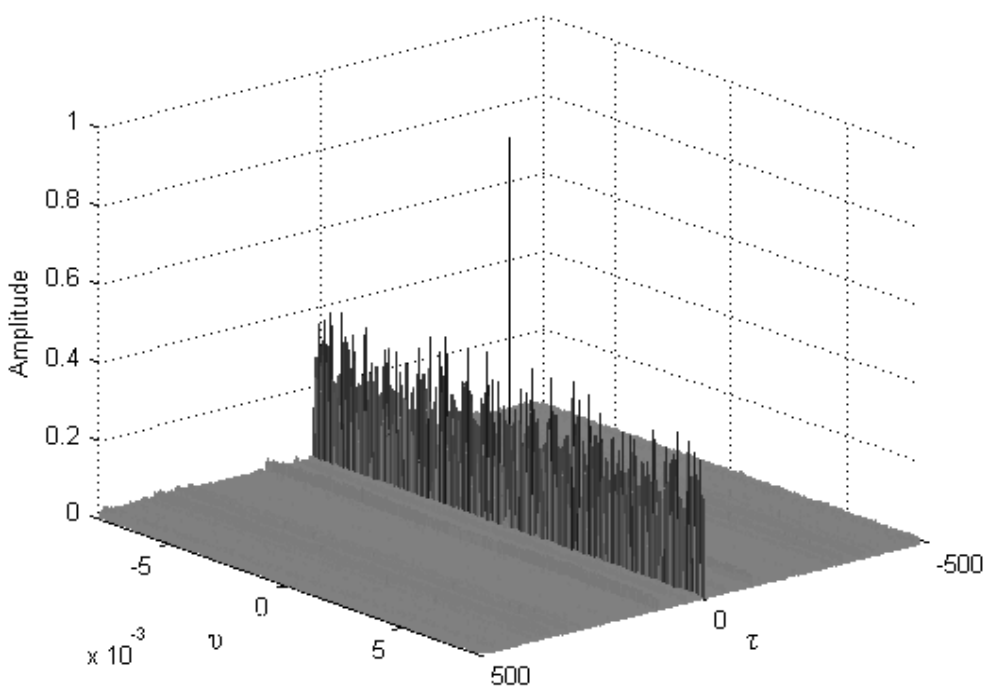

Figure 2. Second-order cyclic statistics of OFDM signal. 


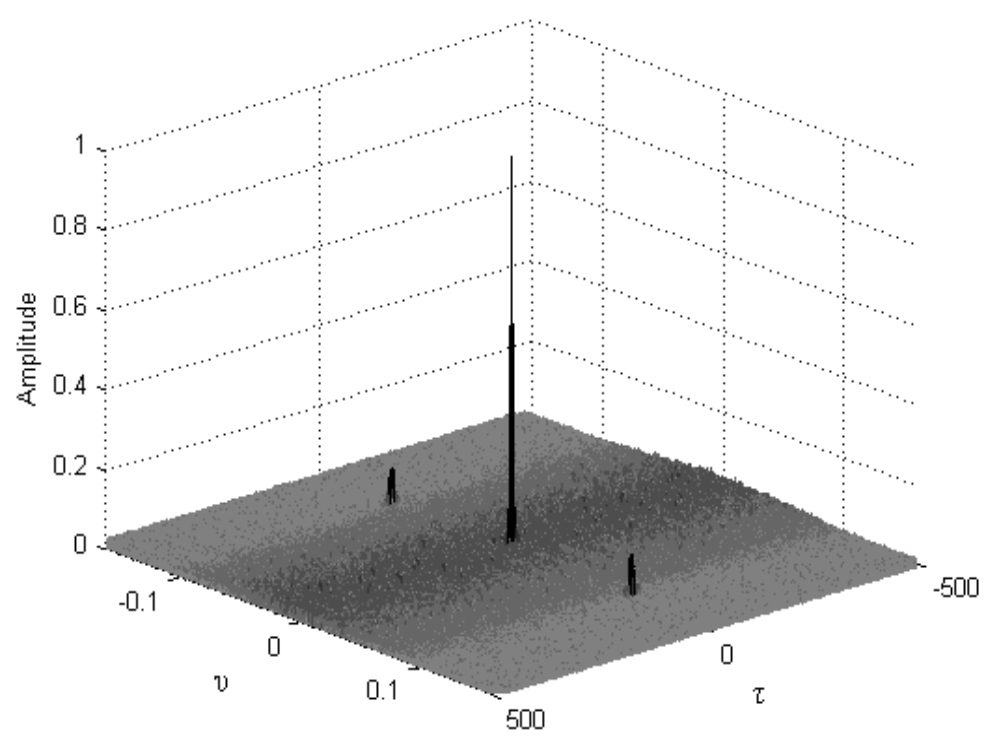

Figure 3. Generalized second-order cyclic statistics of SCLD signal.

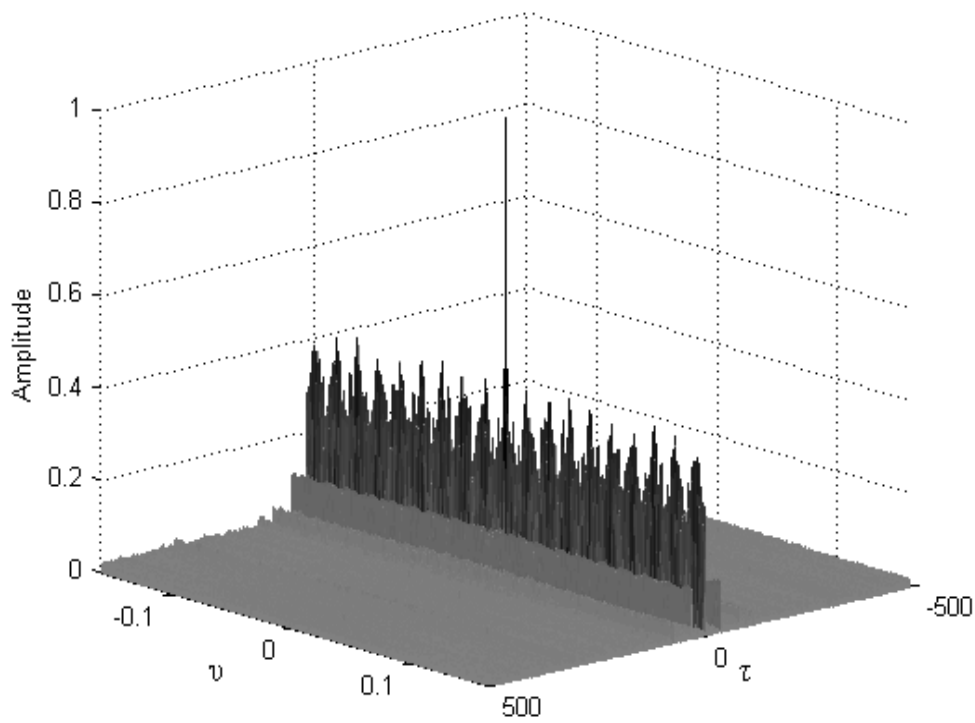

Figure 4. Second-order cyclic statistics of SCLD signal.

the magnitude of the generalized cyclic statistics of the OFDM signal (at $\tilde{\tau}=N_{u}$ and $v=l / N_{s}^{-1}$ ) is employed as discriminating feature to recognize OFDM against SCLD signals.

\section{Performance of proposed recognition algorithm}

Without loss of generality, set $\alpha=1.5 .200$ tests for each MSNR value, and observation interval is set to $0.32 \mathrm{~s}$. The simulation results are shown in Figure 5.

In Figure 5, the probability of correctly recognizing an OFDM signal is plotted versus MSNR. According to Figure 5, the algorithm based on traditional second-order cyclic statistics severely degraded within the range of the entire MSNR, but the proposed algorithm has a significant performance advantage. Note that the probability of correct detection and OFDM signal of the proposed 


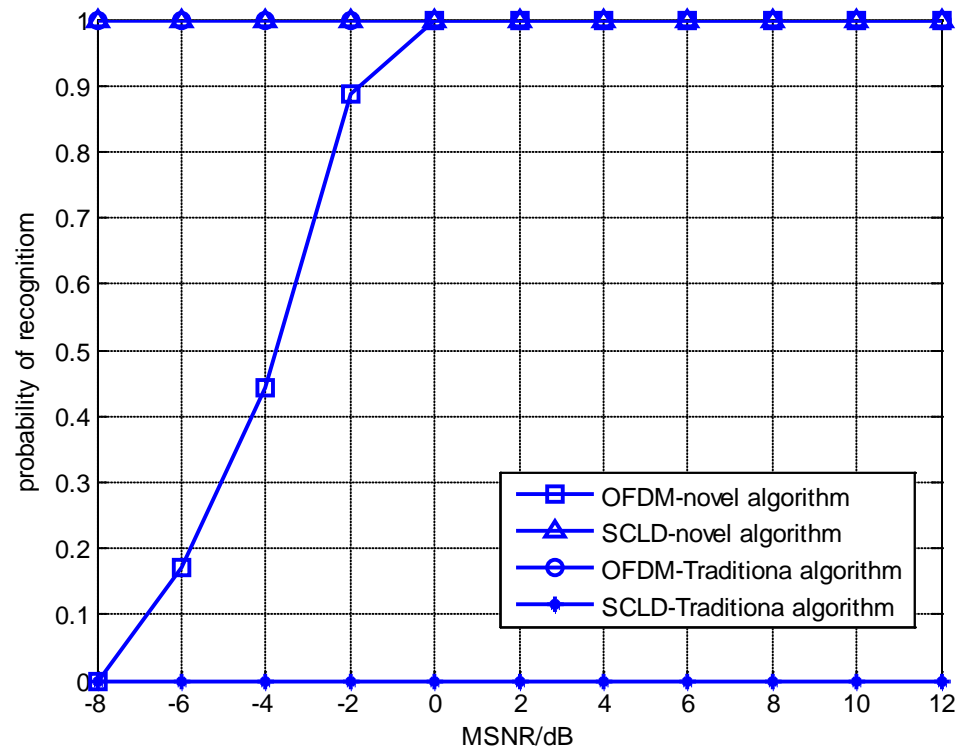

Figure 5. The probability of correct recognition in standard SaS noise.

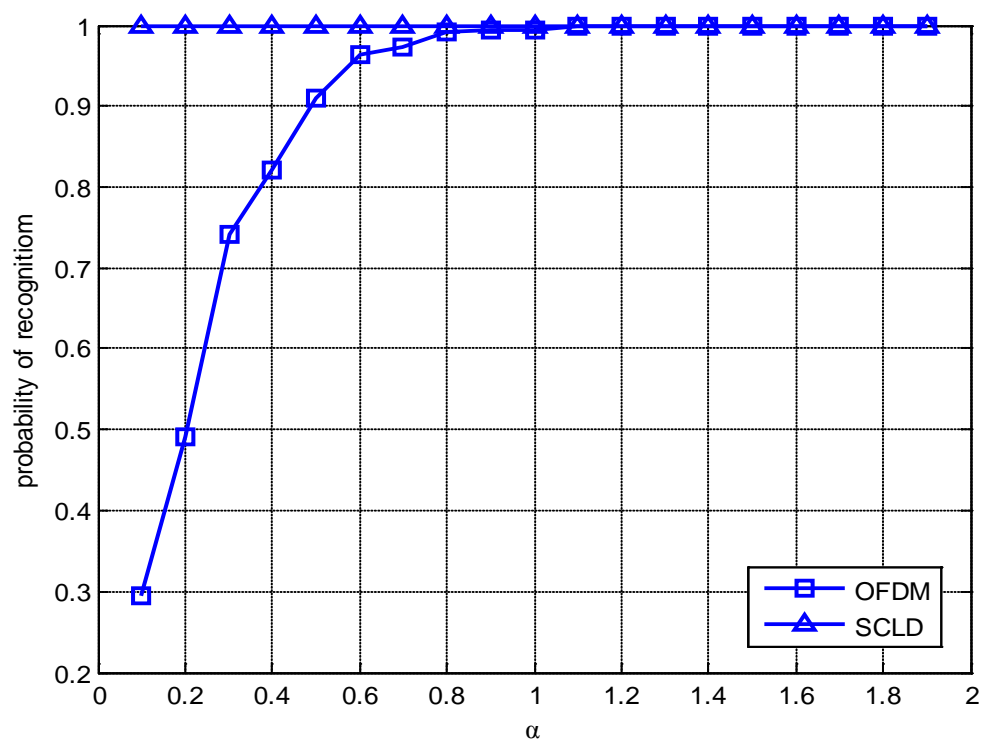

Figure 6. The probability of correct recognition with different values of $\alpha$.

method is almost $100 \%$ at $0 \mathrm{~dB}$. This simulation results for the algorithm performance confirm its effectiveness in alpha- stable distribution noise.

\section{Effect of the $\alpha$ value on algorithm performance}

In this experiment, set MSNR $=0 \mathrm{~dB}, \alpha$ changes with the step 0.1 in the interval $[0.1,1.9]$. The observation interval is set to $0.32 \mathrm{~s}$, and 200 tests for each $\alpha$ value. The simulation results are shown in Figure 6.

Figure 6 shows performance results obtained for different values of $\alpha$ with the proposed algorithm. From Figure 6 as can be seen simultaneously, the smaller the $\alpha$ value, the greater error of correct recognition. The reason is that the smaller $\alpha$ value and the more obvious peak characteristics of alpha-stable distribution. The probability of correct recognition of OFDM is $100 \%$, when 
$\alpha \geq 1$. This simulation result that the proposed method is suitable for different values of $\alpha$ in the interval $[1,2)$.

\section{Conclusion}

This paper proposes a novel modulation identification algorithm for OFDM and SCLD in alpha-stable distributed noise. The generalized second-order cyclic statistics can be exploited for the recognition of OFDM against SCLD signals. The proposed recognition algorithm eliminates the need for preprocessing tasks, such as symbol timing estimation, carrier and waveform recovery, and signal and noise power estimation. Simulation results show that the algorithm have good estimation performance and high robustness.

\section{References}

[1] Grillo, G., Li, H., Bar-Ness, Y., Abdi, A., Somekh, O.S. and Su, W. (2006) OFDM Modulation Classification and Parameter Extraction. Proc. IEEE CROWCOM, 1-6. https://doi.org/10.1109/crowncom.2006.363474

[2] Wang, B. and Ge, L. (2005) A Novel Algorithm for Identification of OFDM Signal. Proc. IEEE WCNC, 261-264.

[3] Meng, L. and Si, X.J. (2007) An Improved Algorithm of Modulation Classification for Digital Communication Signals Based on Wavelet Transform. International Conference on Wavelet Analysis and Pattern Recognition, 1226-1231. https://doi.org/10.1109/ICWAPR.2007.4421621

[4] Zhang, J. and Li, B. (2008) A New Modulation Identification Scheme for OFDM in Multipath Rayleigh Fading Channel. International Symposium on Computer Science and Computational Technology IEEE Computer Society, 793-796. https://doi.org/10.1109/iscsct.2008.192

[5] Punchihewa, A., et al. (2010) On the Cyclostationarity of OFDM and Single Carrier Linearly Digitally Modulated Signals in Time Dispersive Channels: Theoretical Developments and Application. IEEE Transactions on Wireless Communications, 9, 2588-2599. https://doi.org/10.1109/TWC.2010.061510.091080

[6] Zhu, Y., Tian, B., et al. (2010) An OFDM Modulation Recognition Algorithm Based on Spectrum Analysis. IEEE International Conference on Signal Processing, 1557-1560. https://doi.org/10.1109/ICOSP.2010.5656364

[7] Tsihrintzis, G.A. and Nikias, C.L. (1996) Fast Estimation of the Parameters of Alpha-Stable Impulsive Interference. IEEE Transactions on Signal Processing, 44, 1492-1503. https://doi.org/10.1109/78.506614

[8] Qiu, T.S. and Zhang, X.X. (2004) Statistical Signal Processing: Non-Gaussion Signal Processing and Applications. Electronic Industry Press.

[9] Zhao, C.H., Yang, W.C. and Shuang, M.A. (2011) Research on Communication Signal Modulation Recognition Based on the Generalized Second-Order Cyclic Statistics. Journal on Communications, 32, 144-150.

[10] Dandawate, A.V. and Giannakis, G.B. (1994) Statistical Test for Presence of Cyclostationarity. IEEE Transactions on Signal Processing, 42, 2355-2369.

https://doi.org/10.1109/78.317857 
Submit or recommend next manuscript to SCIRP and we will provide best service for you:

Accepting pre-submission inquiries through Email, Facebook, LinkedIn, Twitter, etc. A wide selection of journals (inclusive of 9 subjects, more than 200 journals)

Providing 24-hour high-quality service

User-friendly online submission system

Fair and swift peer-review system

Efficient typesetting and proofreading procedure

Display of the result of downloads and visits, as well as the number of cited articles Maximum dissemination of your research work

Submit your manuscript at: http://papersubmission.scirp.org/

Or contact ijens@scirp.org 